\title{
un/doing clientification: Zur Konstruktion und Dekonstruktion des Klient*innenstatus in der stationären Sozialen Altenarbeit
}

\author{
Grit Höppner
}

Eingegangen: 23. August 2020 / Angenommen: 5. Juni 2021 / Online publiziert: 21. September 2021

(C) Der/die Autor(en) 2021

Zusammenfassung Ohne Klient*innen wäre Soziale Arbeit nicht möglich. Die Bezeichnung „Klient*in“ wirft jedoch die Frage auf, wer oder was genau damit gemeint ist und in welchem Verhältnis Soziale Arbeit zu Klient*innen steht. Dies ist im Handlungsfeld der stationären Sozialen Altenarbeit besonders relevant, in dem ältere Menschen in erster Linie als Bewohner*innen leben und lediglich kontextspezifisch zu Klient*innen werden. Diese fehlende Eindeutigkeit in der Zuschreibung des Klient*innenstatus hat auch zur Folge, dass der Auftrag der Sozialen Arbeit in diesem Handlungsfeld bisher nicht eindeutig definiert ist und die Einrichtung eines Sozialen Dienstes in Seniorenheimen keine Selbstverständlichkeit darstellt. Dieser Beitrag zielt auf eine Präzisierung des Klient*innenstatus in diesem Feld ab. Mit Hilfe der Heuristik des un/doing clientification soll bestimmt werden, wie der Klient*innenstatus konstruiert und dekonstruiert wird und welche Aufgaben der Mitarbeitenden des Sozialen Dienstes damit einhergehen. Dies wird mit Hilfe der Ergebnisse eines ethnographischen Forschungsprojektes veranschaulicht. Es werden all jene sozialen Praktiken dargestellt, in denen der Klient*innenstatus im praktischen Tun hervorgebracht, aufrechterhalten, in Frage gestellt oder irrelevant gemacht wird. Die Ergebnisse ermöglichen neue Impulse für das Verständnis des Klient*innenstatus in der stationären Sozialen Altenarbeit und verdeutlichen die Bedeutung des Sozialen Dienstes in Seniorenheimen.

Schlüsselwörter Klientifizierung · Deklientifizierung · Soziale Praktiken · Sozialer Dienst $\cdot$ Seniorenheim

Unter Mitarbeit von Micha Poser.

Prof. Dr. Grit Höppner $(\bowtie)$

Katholische Hochschule Nordrhein-Westfalen, Piusallee 89, 48147 Münster, Deutschland

E-Mail: g.hoeppner@katho-nrw.de 


\title{
un/doing clientification: on the construction and deconstruction of client status in stationary social work with older people
}

\begin{abstract}
Social work would not be possible without clients; however, the term "client" raises the question of who or what exactly is meant by it and in which relationship social work stands to clients. This is particularly relevant in the field of stationary social work for older people, where people primarily live as residents and only become clients in a context-specific way. This lack of unambiguity in the attribution of client status also has the consequence that the mandate of social work in this field of action has not yet been clearly defined and the establishment of a social service in homes for the aged is not a matter of course. This article aims at specifying the client status in this field. With the help of the idea of un/doing clientification, it is determined how the client status is constructed and deconstructed and which tasks of the social service personnel accompany this. This is illustrated with the results of an ethnographic research project. All those social practices are presented in which the client status is produced, maintained, questioned or made irrelevant in action. The results provide new impulses for the understanding of client status in stationary social work for older people and illustrate the importance of social service in homes for the aged.
\end{abstract}

Keywords: Clientification · Declientification $\cdot$ Social practices $\cdot$ Social service . Homes for the aged

\section{Einleitung}

Ohne Klient*innen ${ }^{1}$ wäre Soziale Arbeit nicht vorstellbar und möglich. Die Arbeit mit Klient*innen stellt ein grundlegendes Ziel und Motiv von Sozialer Arbeit dar, und Klient*innenzentriertheit hat sich als ein selbstverständliches Arbeitsprinzip etabliert. Der Begriff „Klient*in“ wirft jedoch die Frage auf, wer oder was genau damit gemeint ist und in welchem Verhältnis Soziale Arbeit zu Klient*innen steht (Aghamiri und Streck 2018; Juhila et al. 2003). Diese Fragen stellen sich insbesondere im Handlungsfeld der stationären Sozialen Altenarbeit, in dem ältere Menschen in erster Linie als Bewohner*innen leben und lediglich kontextspezifisch zu Klient*innen des Sozialen Dienstes und damit der Sozialen Arbeit werden können. Die fehlende Eindeutigkeit in der Zuschreibung des Klient*innenstatus in diesem Handlungsfeld hat auch zur Folge, dass der Auftrag und die Aufgaben von Sozialer Arbeit bis heute nicht eindeutig definiert sind - anders als dies beispielsweise im Kinderschutz der Fall ist (Streck et al. 2018, S. 256) - und die Einrichtung eines Sozialen Dienstes im Seniorenheim demzufolge keine Selbstverständlichkeit darstellt (Hirt 2008; Schweppe 1996, 2012).

Dieser Beitrag zielt mittels einer Analyse des Klient*innenstatus in Seniorenheimen auf eine Präzisierung, wie Soziale Arbeit im Kontext der stationären Altenarbeit

\footnotetext{
1 Der Begriff Klient*innen wird hier synonym verwendet zu den Begriffen Adressat*innen und Nutzer*innen.
} 
agieren kann und welche Aufgaben Mitarbeitende des Sozialen Dienstes übernehmen. Dabei soll gezeigt werden, dass die Bezeichnung „Klient*in“ keine universale, vorgegebene Kategorie darstellt, sondern dass der Klient*innenstatus in alltäglichen, interaktiven Praktiken situativ und kontextspezifisch hergestellt und aufrechterhalten aber auch in Frage gestellt oder irrelevant gemacht werden kann, und dass damit jeweils bestimmte Aufgaben der Sozialen Arbeit einhergehen. Mit dieser Fokussierung knüpft der Beitrag an eine konstruktivistische Forschungstradition in den Sozialarbeitswissenschaften an, in der danach gefragt wird, wie Soziale Arbeit gemacht wird (Aghamiri et al. 2018; Hall et al. 2003, 2014; für den Bereich des Pflegeheims siehe z. B. die ethnographische Studie von Ammann et al. 2019). So wird etwa im Konzept des Doing Social Work (Aghamiri et al. 2018) davon ausgegangen, dass Soziale Arbeit als eine soziale Wirklichkeit zu verstehen ist, die durch interaktive Konstruktionsleistungen der beteiligten Akteur*innen im gemeinsamen Handeln produziert wird (,doings“). Was in dieser Forschungstradition bisher selten analysiert wurde, ist die Frage nach den Praktiken, in denen Soziale Arbeit oder der Klient*innenstatus im Sinne eines ,undoing“ in Frage gestellt oder zwischenzeitlich irrelevant gemacht wird (als Ausnahme etwa Koch et al. 2018). In diesem Beitrag soll deshalb nicht nur gezeigt werden, wie der Klient*innenstatus im Sinne eines doing clientification hergestellt und aufrechterhalten wird, sondern auch, wie der Klient*innenstatus im Sinne eines undoing clientification abgelehnt, unterlaufen, ausgesetzt oder vergessen werden kann. Um sowohl diese „,doings“ als auch die „undoings“" systematisch darstellen zu können, soll nicht nur der Umgang zwischen Menschen, sondern auch der mit Dingen einbezogen werden. Den Mehrwert, auch Dinge in die Analyse einzubeziehen, verdeutlichen etwa Arbeiten im Bereich der stationären Altenarbeit (Höppner und Brinkmann 2020), im Kontext von institutionellen Gesprächen im Jobcenter (Böringer und Wolff 2012; Schröder 2012) und in der Auseinandersetzung mit digitalen Medien und Informationstechnologien im Rahmen von professionstheoretischen, methodischen und organisationalen Überlegungen in Handlungskontexten der Sozialen Arbeit (Kutscher et al. 2020).

Um dieses Vorhaben umzusetzen, wird zunächst der methodologische Bezugsrahmen zur Rekonstruktion von Klientifizierungs- und Deklientifizierungsprozessen im Sinne eines un/doing clientification entwickelt und dieser anschließend auf ein ethnographisches Forschungsprojekt angewendet, das im Handlungsfeld der stationären Sozialen Altenarbeit durchgeführt wurde. Die Ergebnisse ermöglichen neue Impulse zum Verständnis des Klient*innenstatus in der stationären Sozialen Altenarbeit und verdeutlichen die Bedeutung des Sozialen Dienstes in Seniorenheimen.

\section{2 un/doing clientification: Methodologischer Bezugsrahmen}

Um analysieren zu können, wie der Klient*innenstatus hergestellt, aufrechterhalten, abgelehnt oder unterlaufen werden kann, bieten sich sozialkonstruktivistische Ansätze wie das Doing Social Work (Aghamiri et al. 2018) in Verbindung mit praxis- 
theoretischen Heuristiken wie die der Theorie Sozialer Praktiken (Reckwitz 2003) und der flachen Ontologie (Schatzki 2016) an. ${ }^{2}$

Die unter dem Begriff des Doing Social Work (Aghamiri et al. 2018) zusammengefassten ethnographischen Forschungen zeigen, wie in interaktiven Herstellungsprozessen Handlungsfelder der Sozialen Arbeit als Orte von institutionalisierter Hilfe hervorgebracht und wie sich involvierte Akteur*innen auf der Grundlage spezifischer Wissensbestände die Bezeichnungen „Klient*innen“ und „Professionelle“ gegenseitig zuweisen und darüber Erwartungen beispielsweise zu institutionalisierten Ordnungen transportieren. Doing Social Work verläuft also ähnlich wie das Doing Gender (West und Zimmerman 1987), in dessen Rahmen die Kategorie Geschlecht als interaktive Konstruktionsleistung hervorgebracht wird: Menschen zeigen sich ihr Geschlecht in einem jeweils kontextualisierten Handeln gegenseitig an und bringen in diesen wechselseitigen Bezügen kulturelles Wissen über Weiblichkeit und Männlichkeit zum Ausdruck.

Rebekka Streck und Kolleginnen (2018, S. 238-251) zeigen in ihren Ausarbeitungen zum Doing Social Work, dass Soziale Arbeit in Form von vier Verfahrensweisen hergestellt wird, die alle auch in verschiedenen Handlungsfeldern typisch sind: entscheiden in Ungewissheit, Diffusitäten bespielen, unterschiedliche Differenzkategorien (be)nutzen und Alltägliches disziplinieren. Klient*innen sind an allen vier Verfahrensweisen teilnehmende Akteur*innen (vgl. Aghamiri und Streck 2018, S. 107). Zwar wird bei Ursula Unterkofler und Kolleginnen (2018, S. 8) die Ko-Konstruktion bzw. interaktive Beteiligung von Klient*innen und Sozialarbeitenden hervorgehoben, die gemeinsam sozialpädagogische Situationen hervorbringen. Vorausgesetzt wird dabei allerdings, dass der Klient*innenstatus als solcher bereits vergeben ist.

Dieser Beitrag setzt zeitlich früher an und soll zeigen, wie der Klient*innenstatus hervorgebracht, aufrechterhalten und auch unterlaufen oder vergessen werden kann. Der Analysefokus liegt somit einerseits auf Herstellungsprozessen des Klient*innenstatus, die als doing clientification bezeichnet werden. Dass der Klient*innenstatus in Settings institutionalisierter Hilfe in unterschiedlichsten Situationen hervorgebracht und somit auch empirisch zu beobachten ist, zeigen vor allem konversationsanalytische Studien anhand der Beratung (Bittner 1981; Reitemeier 2000), der Supervision (Meier und Wolff 1997) und von Hilfeplangesprächen in der Kinder- und Jugendhilfe (Meßmer und Hitzler 2007). So hat zum Beispiel Heinz Meßmer (2013) die Kommunikationsmuster der sozialen Kategorisierung, der sozialen Passung, des Redens über/mit und der sozialen Adressierung als „,Klientifizierung“ in Hilfeplangesprächen rekonstruiert, die er als Mechanismen ,zur institutionellen Inklusion“ (ebd., S. 323) deutet.

Wenn der Klient*innenstatus immer wieder aufs Neue performativ herzustellen ist, dann ist davon auszugehen, dass es auch Situationen gibt, in denen der Klient*innenstatus in Frage gestellt, negiert oder vergessen werden kann. Solche Prozesse der Dekonstruktion des Klient*innenstatus werden in diesem Beitrag mit dem

\footnotetext{
2 Der Analysefokus in diesem Beitrag liegt demzufolge auf den „doings“ und ,undoings“ des Klient*innenstatus und nicht auf einer Analyse von Netzwerkbildungsprozessen im Seniorenheim im Sinne der Actor-Network-Theory (vgl. hierzu Heitmann-Möller und Remmers 2017).
} 
Begriff des undoing clientification gefasst. Dieser Begriff ist angelehnt an Ideen zum Undoing Gender, die konzeptionell in zwei Richtungen ausdifferenziert wurden: Judith Butlers (2004) Verständnis von Undoing Gender fokussiert auf individuelle, subversive Praktiken, die sich restriktiven Normen zu Geschlecht und Sexualität widersetzen. Dabei ist sowohl eine kritische Infragestellung von normativen Geschlechterordnungen als auch die Entwicklung von alternativen Herstellungsmodi von Geschlecht zentral (ähnlich auch Deutsch 2007). Hier sind auch die von Meßmer (2013) rekonstruierten Kommunikationsmuster einzuordnen, die er als Prozesse der „Deklientifizierung“ beschreibt, mit denen die ,institutionell[e] Exklusion“ (ebd., S. 329) verfolgt wird. Sie läuten das Ende einer Hilfe ein oder legitimieren das Ende und sind durch die Aufhebung von Interaktionsasymmetrien charakterisiert, die Ist-Soll-Differenzen nivellieren oder die Hilfebeendigung als Konflikt rahmen. Eine zweite Auslegung von Undoing Gender hat Stefan Hirschauer (2001, 2013) entwickelt. Er geht davon aus, dass soziale Praktiken des Doing Gender auch unterbrochen, vergessen oder irrelevant gemacht werden können. Hirschauer (2013) schlägt vor, Geschlechterkonstruktionen und Geschlechterunterscheidungen nicht als omnipräsente Prozesse aufzufassen, sondern deren episodenhaften Charakter anzuerkennen. Geschlecht kann dann stellenweise aus dem Blick geraten, wenn es nicht permanent wiederholt wird: Menschen nehmen sich dann möglicherwiese nicht als weiblich oder männlich wahr und/oder ihr Gegenüber adressiert sie nicht als Frau oder Mann. Dann ruht die Mitgliedschaft als Angehörige einer Geschlechtergruppe so lange, bis ihr Geschlecht wieder relevant gemacht wird. Mit seinem Verständnis von Undoing Gender problematisiert Hirschauer insbesondere die Infrastrukturen von Geschlecht, die Möglichkeiten schaffen, Geschlecht zwischenzeitlich irrelevant zu machen.

Um die Ko-Konstitution unterschiedlicher Akteur*innen im Vollzug eines un/ doing clientification konsequent zu berücksichtigen, soll in der folgenden Analyse danach gefragt werden, welche Akteur*innen in den Prozess der Herstellung des Klient*innenstatus involviert sind, was diese Akteur*innen gemeinsam tun und welches Wissen über Klient*innen in diesem Tun aktualisiert wird. Mit diesen Fragestellungen werden die bisher herausgearbeiteten konzeptionellen Bezüge um praxistheoretische Ideen ergänzt. Trotz ihrer vielfältigen Ausarbeitungen eint Praxistheorien die zentrale Frage danach, wie sich soziale Ordnungen im praktischen Zusammenspiel von Körpern und Artefakten erzeugen und verändern und wie sich dadurch Wissensordnungen und Routinen konstituieren und verfestigen (vgl. Schatzki 2002; Schmidt 2012). Praxistheorien fokussieren auf soziale Praktiken als zentrale Kategorien, die als „kleinste Einheit des Sozialen“ (Reckwitz 2003, S. 290) verstanden werden. Als Träger von sozialen Praktiken bestimmt Andreas Reckwitz zum einen Körper, denn soziale Praktiken seien ,,skillful performances von kompetenten Körpern“ (ebd.). Solch eine Performance zeigt sich sowohl „nach innen“ durch das Verinnerlichen von Knowhow bzw. einem praktischen Verstehen, als auch „nach außen“ durch motorische Aktivitäten, die für andere sichtbar sind. Als weiteren Bestandteil von sozialen Praktiken nennt Reckwitz Artefakte wie ein Buch oder einen Computer. Folglich umfasst die Materialität sozialer Praktiken gleichermaßen Körper und Artefakte. Diese werden durch ein gemeinsames Tun zusammengebracht und zusammengehalten. Diese gemeinsame Performance beschreibt Theodore R. Schatzki 
(2002, S. 71) als „organisierte Aktivitäten“, die sich aus offenen, raum-zeitlich verteilten Mengen von ,doings and sayings“ zusammensetzen, die wiederum durch ,gemeinsame Verständnisse, Teleoaffektivität (Zwecke, Ziele, Emotionen) und Regeln organisiert [sind]" (Schatzki 2016, S. 33). Hierbei spielt auch die Implizitheit von Wissen (Reckwitz 2003; Schatzki 2002) eine wichtige Rolle. Praktisches Verstehen umfasst nach Schatzki einerseits ein implizites Wissen zu „doings and sayings“ und das entsprechende Knowhow, diese auszuführen. Andererseits beinhaltet praktisches Verstehen auch die Fähigkeit, ,doings and sayings“ als solche zu erkennen.

Als methodologische Konsequenz für die folgende Analyse ergibt sich ein auf soziale Praktiken hin ausgerichteter Fokus. Es wird angenommen, dass sich sowohl Prozesse der Konstruktion als auch der Dekonstruktion des Klient*innenstatus in sozialen Praktiken abbilden lassen. Dabei interessiert, welche Menschen, Alltagsgegenstände, technischen Artefakten und Räumlichkeiten in die Konstruktion und Dekonstruktion des Klient*innenstatus eingebunden werden und wie ihr Zusammenspiel organisiert ist. Mit diesen Fragen ist eine konsequente analytische Blickwinkelverschiebung von Individuen als zentrale Akteur*innen der Konstruktion und Dekonstruktion des Klient*innenstatus hin zu den multiplen Teilnehmenden verbunden, die an diesen Prozessen beteiligt sind. Deren gemeinsame Performance in sozialen Praktiken kann in Form von Beobachtungen protokolliert und durch Interviewtranskripte erfasst werden. In der Analyse des empirischen Materials soll schließlich das Wissen rekonstruiert werden, das Rückschlüsse auf bestimmte Bedeutungen, soziale Institutionen, historische Entwicklungen und kulturelle Muster ermöglicht, die in Praktiken transportiert werden (vgl. Schäfer 2016). Zu fragen ist in der folgenden Analyse deshalb auch, welches kulturelle Wissen in Klientifizierungs- und Deklientifizierungsprozessen hervorgebracht wird. Dabei soll gezeigt werden, dass soziale Praktiken in ein ,field of practices“ (Schatzki 2002, S. 11) eingebettet sind, eine Praktik also nicht für sich allein steht, sondern erst in ihrer Verwobenheit mit anderen sozialen Praktiken erfasst werden kann. Hier zeigt sich noch einmal die Relevanz, Vorstellungen zum Klient*innenstatus in der stationären Sozialen Altenarbeit nicht allein durch eine Betrachtung des doing clientification herauszuarbeiten, sondern auch durch eine Betrachtung des undoing clientification.

\section{Forschungsdesign}

Das Forschungsprojekt, das im Folgenden vorgestellt wird, bezieht sich auf das Handlungsfeld der stationären Sozialen Altenarbeit. Die untersuchten Seniorenheime verfügen alle über einen Sozialen Dienst, in dem jeweils ein bis zwei Sozialarbeitende und mehrere Betreuungsassistent*innen ${ }^{3}$ eng zusammenarbeiten. Nach Kontaktaufnahmen per E-Mail und Telefon wurden im Rahmen eines ethnographischen Vorgehens in verschiedenen Seniorenheimen in Städten in Nordrhein-Westfalen vier

\footnotetext{
3 Die Betreuungsassistent*innen sind durch ein Studium der Sozialen Arbeit an einer Hochschule so qualifiziert wie die Sozialarbeitenden und verfügen meist über langjährige Berufserfahrungen in diesem Feld. Aus diesem Grund werden die Aussagen aller interviewten Mitarbeitenden des Sozialen Dienstes im Folgenden gemeinsam dargestellt.
} 
teilnehmende Beobachtungen von Gruppenangeboten der Sozialen Altenarbeit zum Gedächtnis- und Bewegungstraining (vgl. Breidenstein et al. 2013), zwei Walking Interviews mit Sozialarbeitenden (vgl. Ratzenböck 2017) und drei problemzentrierte Interviews mit Sozialarbeitenden und Betreuungsassistent*innen (vgl. Witzel 2000) durchgeführt. Um sowohl das doing clientification als auch das undoing clientification abzubilden, wurden die Passagen aus den Interviewtranskripten und Beobachtungsprotokollen analysiert, in denen die Mitarbeitenden des Sozialen Dienstes den Klient*innenstatus relevant gemacht oder Verweigerungen oder Irrelevantsetzungen des Klient*innenstatus durch die Bewohner*innen zum Ausdruck gebracht haben. Bezüglich der Datenauswertung stützt sich das Forschungsprojekt auf das Kodierverfahren als ein wesentliches Merkmal der Grounded Theory (vgl. Strauss 1998). Das Kodierverfahren dient der systematischen und thematischen Aufschlüsselung und Sortierung des transkribierten empirischen Materials; mit ihm sollten zentrale Kategorien (,Schlüsselkategorien“) generiert werden, die soziale Praktiken von doing clientification und undoing clientification abbilden. Diese Praktiken werden im Folgenden vorgestellt.

\section{4 un/doing clientification: Praktiken der Konstruktion und Dekonstruktion des Klient*innenstatus}

Bevor Prozesse des un/doing clientification in einem Seniorenheim ablaufen können, erfolgen Tätigkeiten wie die Kontaktaufnahme durch Senior*innen oder deren Angehörige, Informationsvermittlung und Überprüfung der Einzugsvoraussetzungen wie dem Pflegegrad und der finanziellen Absicherung. ${ }^{4}$ Münden diese Tätigkeiten in die Entscheidung zum Einzug in ein Seniorenheim, beginnen Prozesse des un/doing clientification. Abb. 1 dient der Veranschaulichung dieser Prozesse. Die analysierten Praktiken der Konstruktion und Dekonstruktion des Klient*innenstatus sind in zeitlicher Hinsicht angeordnet.

\section{1 doing clientification}

Die Konstruktion des Klient*innenstatus ist untrennbar mit einer zeitlichen Einordnung verbunden. Senior*innen, die in ein Seniorenheim einziehen, werden unmittelbar zu offiziellen Bewohner*innen und zugleich auch zu Klient*innen der Sozialen Arbeit: ,der Bewohner wird [...] begleitet beim Einzug, auch vom sozia-

\footnotetext{
${ }^{4}$ Häufig ist diese Phase durch ein vorangegangenes Ereignis wie eine akute gesundheitliche Einschränkung gekennzeichnet, das den Umzug eines bis dato (weitgehend) autonomen älteren Menschen in ein Seniorenheim erst erforderlich macht (vgl. Backes und Clemens 2013, S. 263). Diese Senior*innen können folglich nicht mehr oder nur bedingt Träger*innen der Praktiken der alltäglichen Selbstsorge sein, die das Einkaufen, Kochen und Hygienehandlungen umfassen, was unterstützende Hilfe notwendig macht. Um in ein Seniorenheim einziehen zu können, müssen Senior*innen (oder ihre Vertreter*innen) ihren Hilfebedarf im Seniorenheim anmelden. Zugleich sind die Mitarbeitenden des Seniorenheims auf solche Anfragen angewiesen, um die Plätze im Seniorenheim zu belegen. Hier besteht eine wechselseitige Abhängigkeit.
} 


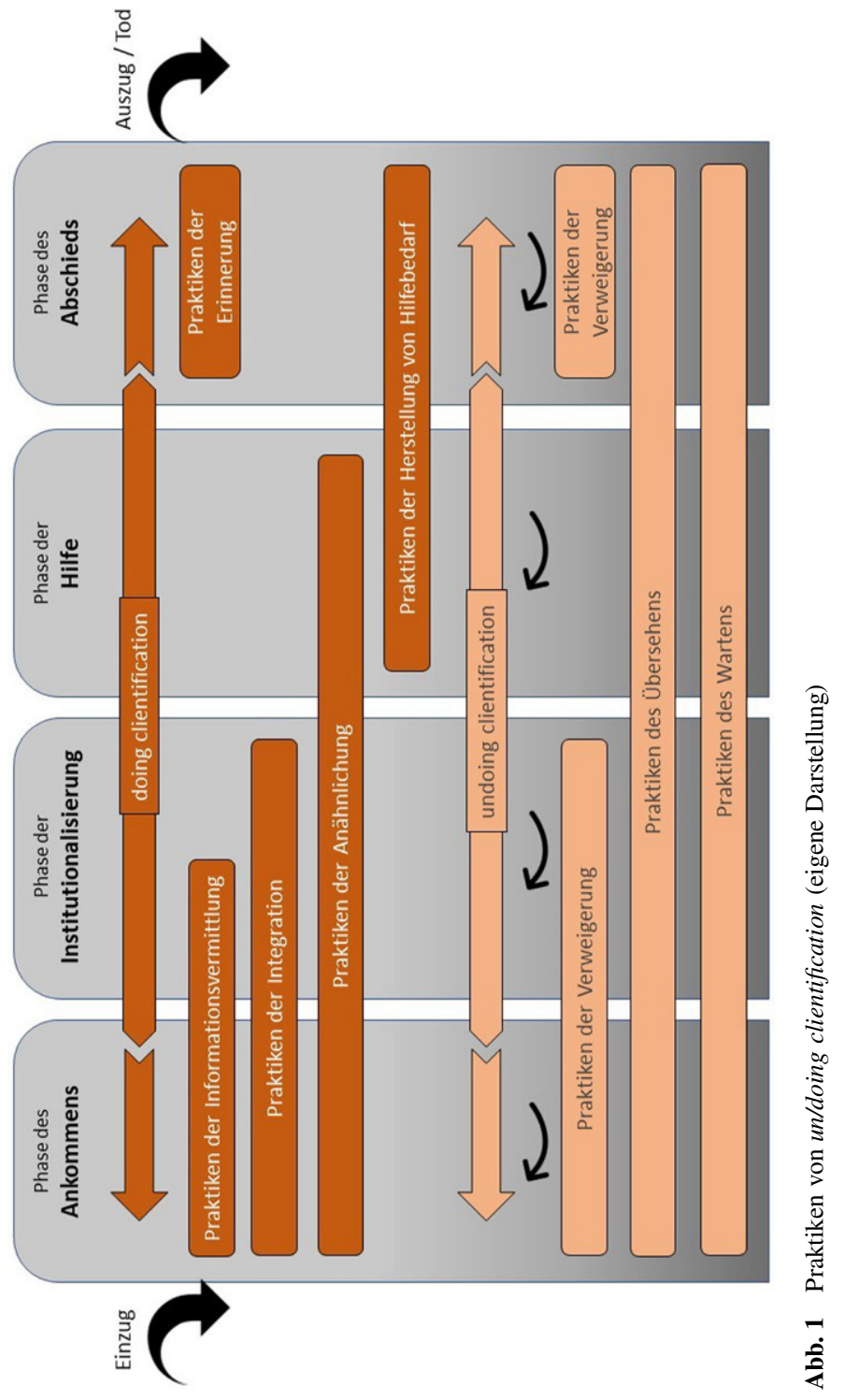


len Dienst. das ist einfach auch notwendig um diese ganzen, die ganzen Rahmenbedingungen zu klär'n“" (Mitarbeiterin Frau Sander ${ }^{5}$ ). Nach dieser institutionellen Einbindung, die insbesondere durch die Gesetzesgrundlage, den MDK und Hausregeln kontextualisiert ist, relativiert sich diese Verknüpfung von Bewohner*innenund Klient*innenstatus wieder: Bewohner*innen des Seniorenheims sind nun nicht mehr automatisch auch als Klient*innen der Sozialen Arbeit zu verstehen, wenngleich prinzipiell jederzeit die Möglichkeit besteht, dass die Mitarbeitenden den Bewohner*innen den Klient*innenstatus wieder zuschreiben. Denn Soziale Arbeit tritt im Seniorenheim nicht als externe Hilfeinstitution auf, sondern ist räumlich und organisatorisch eng verzahnt mit den alltagsweltlichen Zusammenhängen der Bewohner*innen - ein Kontakt zum Sozialen Dienst ist somit fast immer unmittelbar möglich.

Praktiken der Herstellung des Klient*innenstatus sind gerade während der ersten Wochen nach dem Einzug an das Prinzip der Freiwilligkeit gebunden. So erzählt eine Mitarbeiterin des Sozialen Dienstes:

„Ich glaube, dass der alte Mensch erst zum Fall der sozialen Altenarbeit im Heim wird, wenn er es auch selber möchte, weil, der soziale Dienst kommt dahin wenn die Person einzieht und schlägt Sachen vor. Sagt das is' n Tagesplan, sie können zu der Veranstaltung oder zu der Gruppe mit. Wir können mal zusammen raus geh'n, oder zusammen nen Kaffee trinken." (Mitarbeiterin Frau Sander)

Nach dem Einzug suchen Mitarbeitende des Sozialen Dienstes die neu eingezogenen Bewohner*innen auf. Dabei steht die Erläuterung der Angebote des Sozialen Dienstes im Vordergrund. An diesen Praktiken der Informationsvermittlung partizipieren Mitarbeitende des Sozialen Dienstes und Artefakte wie ein Tagesplan zur Veranschaulichung der täglichen Struktur, die über die lebenserhaltene Struktur im Seniorenheim (wie Mahlzeiten, Tablettengabe) hinausgeht. Diese Praktiken verlaufen idealtypisch, wenn sich Bewohner*innen als Teilnehmende an diesen Praktiken rekurrieren lassen; nur dann können Informationen vermittelt und Aktivitäten angegangen werden, wie die Teilnahme an Veranstaltungen, Gruppenangeboten, Spaziergängen, gemeinsames Kaffeetrinken. Praktiken der Informationsvermittlung sind wechselseitig ausgerichtet: Während der Soziale Dienst über seine Möglichkeiten im Seniorenheim informiert und sich eine Zusammenarbeit mit den neuen Bewohner*innen erhofft, vermitteln die Bewohner*innen durch ihr Interesse oder Desinteresse an Angeboten ihre Wünsche und Erwartungen.

In Praktiken der Informationsvermittlung werden am institutionellen Ablauf und Angebot interessierte Klient*innen konstruiert, die für die Kommunikation mit anderen Menschen offen sind und durch verschiedene Aktivitäten anstreben, sich in die Gemeinschaft im Seniorenheim zu integrieren. Oft ist zu diesem Zeitpunkt noch kein klarer individuumsbezogener Auftrag für die Soziale Arbeit abzuleiten, und die Form der Unterstützung ist noch relativ unbestimmt.

Parallel oder zeitlich etwas versetzt zu dieser Phase des Ankommens, die in ihrer Länge variieren kann, verläuft die Phase der Institutionalisierung. Hier sind zum

\footnotetext{
5 Die Namen der Interviewten wurden anonymisiert.
} 
einen Praktiken der Integration in die Gemeinschaft angesprochen. Diese Praktiken zeichnen sich insbesondere durch die Initiative der Mitarbeitenden des Sozialen Dienstes aus. Einerseits unterstützen sie die Integration der neuen Bewohner*innen durch „feste Tischgemeinschaften“ im Gegensatz zur freien Platzwahl im Essensraum, die vom Sozialen Dienst durch die Stellung von Tischen und Stühlen vorstrukturiert und personell zusammengesetzt werden, wenn ein „Platz frei [war] wo man dachte, dass passt gut vielleicht oder es sind zwei Damen, wo man denkt die versteh'n sich vielleicht ganz gut." (Mitarbeiterin Frau Sander). Andererseits kontaktieren die Mitarbeitenden des Sozialen Dienstes die Bewohner*innen, und werden im Idealfall von diesen willkommen geheißen oder schon erwartet (,Schön, dass sie mal wieder vorbei komm“) und deren Angebote angenommen (,Nehm“ sie mich mit zu der Veranstaltung, ich würd da gerne hin, mich interessiert das"). Die Mitarbeitenden des Sozialen Dienstes beobachten den Fortgang der Integration der Bewohner*innen und schlussfolgern, dass Bewohner*innen angekommen sind, wenn sie entweder sagen "Ich fühl mich hier wohl“" und ,das Essen hat heute gut geschmeckt“ oder wenn sie sehen, dass die Bewohner*innen ,irgendwie präsent“ sind, dann ,läuft [sie oder er] mal durch den Flur mit dem Rollator, mal rauf und runter, um sich ein bisschen zu bewegen oder zu gucken, was so auf dem Flur los ist oder fragt mal nach, wann gibt's denn heute Kaffeetrinken oder geht auf andere Bewohner zu“ (Mitarbeiterin Frau Sander).

Zum anderen sind in dieser Phase Praktiken der Anähnlichung angesprochen, die für andere visuell wahrnehmbar sind. Damit ist erstens das zur Verfügungstellen von Möbeln bei spärlicher Einrichtung aufgrund der fehlenden Nähe von Angehörigen durch den Sozialen Dienst gemeint, damit das Zimmer ,gut eingerichtet ist" (vgl. genauer Depner 2015). Zweitens beziehen sich diese Praktiken auf das zur Verfügungstellen eines Rollators durch den Sozialen Dienst für all jene Bewohner*innen, die noch keinen Rollator besitzen (Mitarbeiterin Frau Sander). Dabei geht es zwar in erster Linie um die Herstellung von Mobilität der Bewohner*innen; in der Konsequenz führt die Ausstattung mit einem Rollator aber zu einer Anähnlichung an andere Bewohner*innen mit Rollator. Diese Anähnlichung drückt sich sowohl in der Nutzung dieses technischen Artefakts aus, die durch eine ähnliche Körperhaltung beim Gehen und eine ähnliche Gehgeschwindigkeit deutlich wird, als auch durch ähnliche Farben (grau, schwarz). Praktiken der Anähnlichung zeigen sich drittens in der räumlichen Nutzung des Seniorenheims, zum Beispiel während der Mahlzeiten. In einem der untersuchten Seniorenheime essen Bewohner*innen mit Orientierung im Essensbereich im Erdgeschoss, während Bewohner*innen mit eingeschränkter Orientierung im Wohnzimmer essen, das auf jeder Etage für die dort lebenden Bewohner*innen zur Verfügung steht. Die Bewohner*innen, die im Wohnzimmer essen, werden von Betreuungassistent*innen beim Essen unterstützt und zum Gespräch animiert. Alle Bewohner*innen mit eingeschränkter Orientierung werden während der Mahlzeiten und wegen der damit zusammenhängenden räumlichen Verortung also zwangsläufig zu Klient*innen der Sozialen Arbeit.

Hier werden Klient*innen konstruiert, die sich in die Gemeinschaft im Seniorenheim integrieren wollen und dies auch weitestgehend selbstständig können, indem sie Angebote des Sozialen Dienstes in Anspruch nehmen, zur Verfügung gestellte Alltagsgegenstände oder technische Artefakte nutzen, das Seniorenheim erkunden 
und Kontakt zu anderen Menschen suchen. Dabei bewegen sie sich im Rahmen der Hausregeln bzw. der Erwartungen und der Etikette im Seniorenheim. Der Auftrag für die Soziale Arbeit ist nun insofern spezifischer als bekannt ist, dass die Integration in das Seniorenheim von Seiten dieser Bewohner*innen erwünscht und realisierbar ist. Bekannt ist auch, dass stellenweise eine Unterstützung durch den Sozialen Dienst in Form von Menschen oder dinghaften und räumlichen Komponenten notwendig sein kann. Auch wenn das Prinzip der Freiwilligkeit weiterhin aktiv ist, so hat die Nutzung von Angeboten des Sozialen Dienstes doch auch einen verbindlicheren Charakter angenommen; institutionelle Strukturen zu kennen und sich in diesen normgerecht zu bewegen, kennzeichnen folglich diese Praktiken.

Eine weitere Phase der Klientifizierung tritt dann ein, wenn Bewohner*innen zunehmend auf Hilfe angewiesen sind, diese erwarten und annehmen. In Praktiken der Herstellung von Hilfebedarf sind ebenfalls nicht allein Bewohner*innen involviert, sondern in noch größerem Umfang als in den zuvor beschriebenen Praktiken Mitarbeitende des Sozialen Dienstes und der Pflege, Angehörige, Alltagsgegenstände und technische Artefakte. Es erfolgt quasi eine Ausweitung der Teilnehmenden an den Praktiken in quantitativer Hinsicht und eine Intensivierung von deren Zusammenarbeit wird antizipiert. Während in Praktiken der Anähnlichung Unterstützung in Form von technischen Artfakten im Sinne der Erleichterung des Gehens gerne angenommen werden, ist in Praktiken der Herstellung von Hilfebedarf die Abwesenheit eines Rollators undenkbar. Dann stehen einige Bewohner*innen ,gar nicht erst [auf], weil sie diese Stütze und die Sicherheit von dem Rollator gar nicht [haben]“ oder sie weichen an einem ,schlechten Tag“ vom Rollator auf den Rollstuhl aus und intensivieren dadurch den Grad an Unterstützung durch technische Artefakte (Mitarbeiterin Frau Sander). Die Aufgabe des Sozialen Dienstes wird darin gesehen, Rahmenbedingungen zu schaffen, um ein Sicherheitsgefühl auf Seiten der Bewohner*innen herzustellen, das es ermöglicht, das eigene Zimmer zu verlassen. Dazu zählen die Bereitstellung eines Rollstuhls und die Vermittlung von Informationen zur Sturzprophylaxe durch Hinweise auf Stolperfallen, Griffe zum Festhalten und Ruhebänke. Hierdurch werden die jeweils adressierten Bewohner*innen zu Klienten*innen der Sozialen Arbeit.

Weitere Praktiken der Herstellung von Hilfebedarf beziehen sich auf die individuelle Förderung einzelner Bewohner*innen, die entweder durch die freiwillige Inanspruchnahme der für sie bereitgestellten Angebote - etwa zur psychosozialen Stabilisierung nach dem Verlust von Angehörigen oder mittels der Biografiearbeit mit Biografiebögen bei Demenz - zu Klient*innen werden. Oder Bewohner*innen werden durch individuell-regulierende Maßnahmen durch die Mitarbeitenden des Sozialen Dienstes zu Klient*innen, wie im Fall einer Bewohnerin, die durch dauerhaftes aggressives Verhalten (d.h. Beschimpfen, mehrstündiges Schreien, Anspucken, Schlagen) auffällt. Ziel ist es dann, dass man ,versucht, dass man da als Sozialarbeit dann interveniert, versucht diese Person zu beruhigen, mit der Person raus zu gehen oder versuchen mit ihr ein Gespräch zu suchen, abzulenken“, um andere Bewohner*innen zu entlasten, die dadurch entstandene Unruhe auszugleichen und dafür anfällige Bewohner*innen nicht zu nicht-normkonformen Verhalten zu triggern. In diesen Fällen spielt „Freiwilligkeit gar nicht mehr so die Rolle, sondern da muss dann (.) interveniert werden, ne“ (Mitarbeiterin Frau Sander). Diese Menschen 
werden als Klient*innen konstruiert, die nur durch die intensive Begleitung durch Mitarbeitende des Sozialen Dienstes in die Gemeinschaft integriert werden können; das Prinzip der Freiwilligkeit ist zum Prinzip der Verpflichtung transformiert. Der Auftrag der Sozialen Arbeit bezieht sich dann auf die Herstellung eines normkonformeren Verhaltens der Bewohner*innen, auf die Ausbalancierung der Stimmung in der Gemeinschaft und auf die Sicherstellung des institutionellen Ablaufs.

Die letzte Phase des Abschieds ist durch die Praktik der wertschätzenden Erinnerung gekennzeichnet. Diese Praktik aktualisiert insbesondere das Wissen um geschätzte Gegenstände, das zuvor im Rahmen der Biografiearbeit generiert wurde:

„Und dann war das letztendlich auch, durch seine äh Grunderkrankung bedingt, so, dass er immer weiter kognitiv abbaute. Und dann brauchte ich letztendlich nur einen diese Modellautos, in die Hand nehm, und dann sprach er mit mir wieder über dieses Auto. Und versuchte es noch zu verbalisieren. Und letztendlich haben wir dann ne Ebene miteinander gefunden in seiner äh finalen Sterbephase. Wo viel auch über taktil Berührung ging. Und wo nochmal wichtig war, diese Modellautos in der Hand zu halten, zu präsentier'n, zu dreh'n, sich anzuschau'n, mir wieder zu zeigen.“ (Mitarbeiter Herr Müller)

Die Praktik der wertschätzenden Erinnerung umfasst eher ,doings“ als „,sayings“, wobei sich „doings“ auf ein ruhiges Erspüren und Wahrnehmen von bekannten Formen bezieht, was das Sicherheits- und Wohlbefinden erhöhen könnte.

In diesem Beispiel wird ein Klient konstruiert, der nicht mehr in der Lage ist, eigene Impulse für Gespräche mit dem Mitarbeiter des Sozialen Dienstes zu setzen, der die Impulse des Mitarbeiters aber aktiv aufnimmt und auf diese reagiert. In dieser Praktik ist das Prinzip der Verpflichtung wieder zum Prinzip der Freiwilligkeit transformiert. Auffallend ist, dass lediglich ein Mitarbeiter des Sozialen Dienstes von dieser Phase des Abschieds berichtete. Damit bleibt offen, ob in dieser Phase der Zuständigkeitsbereich des Sozialen Dienstes gleichermaßen wie in anderen Phasen tangiert ist.

\section{2 undoing clientification}

Prozesse der Dekonstruktion des Klient*innenstatus sind zeitlich weniger eindeutig wie Prozesse der Konstruktion des Klient*innenstatus. Sie zeigen sich zum einen im Sinne von Butlers und Deutschs Verständnis der subversiven Praktiken, die sich dem normativen Bezugsrahmen im Seniorenheim widersetzen.

Die Praktiken der Verweigerung erläutert die Mitarbeiterin Frau Sander am Beispiel eines Mannes, der seit einiger Zeit im Seniorenheim lebt:

,[Er] saß eigentlich nur im Zimmer und hat Fernseh'n geguckt und ich bin jeden Tag rein, hab immer wieder gesagt, wollen sie nicht mit raus komm, und er hat jeden Morgen ist er zum äh Frühstück gegangen und hat danach an der Rezeption seine Zeitung abgeholt (.) Das war sein Gang und dann ging es auf sein Zimmer und dann (.) hat er den ganzen Tag Zeitung gelesen bis es dann Mittagessen gab und dann danach Fernsehn geguckt, und der war wirklich (.) nie mit dabei. Und hat aber immer gesagt, es wäre alles ok, er würde sich wohl fühl'n.“ 
Dieser Mann ist als Bewohner im Seniorenheim angekommen, weist aus der Sicht der Mitarbeiterin durch die Verweigerung der Teilnahme an Angeboten des Sozialen Dienstes den Klient*innenstatus aber konsequent zurück. Explizit stellt er nicht die Angebote bzw. die institutionellen Abläufe und die Gemeinschaft infrage, wohl aber entwickelt er eine Alternative zum Klientenstatus, indem er ausschließlich den Bewohnerstatus aufrechterhält. Praktiken der Verweigerung sind durch Beständigkeit und Vehemenz gekennzeichnet und auch durch einen räumlichen Rückzug in das private Zimmer, mit der Konsequenz, nicht an der Gemeinschaft zu partizipieren und auf der institutionellen Ebene der Sozialen Arbeit exkludiert zu sein. Dass Praktiken der Verweigerung im Seniorenheim überhaupt ausführbar sind, bedarf einer expliziten Begründung durch die Mitarbeiterin, die sie im Fall des Mannes in seiner starken Trauer um die verstorbene Ehefrau ausmacht.

Hier wird ein Bewohner konstruiert, der aufgrund der Trauer um seine verstorbene Ehefrau eigensinnig und vor allem wirksam die Teilnahme an Angeboten des Sozialen Dienstes ablehnt. Anders als in den von Meßmer (2013, S. 329) rekonstruierten Kommunikationsmustern zur ,Deklientifizierung als Mechanismus institutioneller Exklusion“ läuten diese Praktiken der Verweigerung aber nicht das Ende einer Hilfe ein. Stattdessen erscheint aus der Sicht der Mitarbeiterin die Hilfe sogar umso dringlicher zu sein, um eine soziale Isolation zu vermeiden. So sucht die Mitarbeiterin den Mann tagtäglich auf und erhofft sich jeden Tag aufs Neue eine Annahme ihres (freiwilligen) Angebotes. Damit festigt sie die Differenz zwischen dem IstZustand (Ablehnung des Angebotes durch den Bewohner) und dem von ihr erwünschten Soll-Zustand (Annahme des Angebotes durch den Bewohner) und macht dadurch deutlich, dass ihre Adressierung des Bewohners als Klient noch keinen Klient*innenstatus schafft; erst die Annahme der Adressierung durch den Bewohner würde den Klient*innenstatus als solchen bestätigen. Weil diese Übereinstimmung aber nicht erfolgt, entfaltet sich ein gewisses Konfliktpotenzial. Zeitlich zu verorten sind diese Praktiken im empirischen Material in den Phasen des Ankommens und der Institutionalisierung, aber sie sind auch in der Phase des Abschieds denkbar, z. B. wenn sozialpädagogische Angebote der wertschätzenden Erinnerung abgelehnt werden.

Der Konterpart zu solchen subversiven Praktiken der Verweigerung wären Praktiken, die durch eine konsequente Zurückweisung der Adressierung als Mitarbeitende gekennzeichnet wären. Diese Praktiken entsprechen allerdings nicht dem Auftrag der Sozialen Arbeit zur Hilfeleistung und sind im Datenmaterial nicht zu finden. Allerdings finden sich im Material Praktiken, durch die ein Hilfebedarf übersehen werden kann. Undoing clientification erfolgt dann nicht im Sinne von Butlers und Deutschs Verständnis von ,undoing“, sondern im Sinne von Hirschauers Überlegungen. Hier sind insbesondere die Infrastrukturen des Seniorenheims von Interesse, die es ermöglichen, die Herstellung bzw. Aufrechterhaltung des Klient*innenstatus zwischenzeitlich zu vergessen oder irrelevant zu machen. So erzählt die Mitarbeiterin Frau Sander, dass Bewohner*innen, die

,auch sozialarbeiterische Hilfe benötigen aber viel stiller sind und sich in ihrem Zimmer zurücksuchen äh zurück äh = ähm verstecken sag ich mal oder zurückziehen ähm dann manchmal vielleicht auch gar nicht so gehört werden [...] aber 
dass die Bewohner es eigentlich auch nötig hätten. Und ähm (.) Ja das glaub ich schon, dass grade Bewohner, die sehr präsent sind (.) und vielleicht auch, ja sag ich mal, im Negativen, sehr präsent sind, laut sind, aggressiv sind (.) auch vermehrt zu Fällen werden, der Sozialen Arbeit. Glaub ich schon, ja.“

Hier wird eine Differenzierung zwischen Bewohner*innen vorgenommen in jene, die durch ihr Verhalten auffallen und dadurch eher zu Klient*innen werden, und in jene, die sich still und zurückgezogen in ihren Zimmern aufhalten, Hilfe nicht explizit einfordern und daher nicht immer auf dem Radar des Sozialen Dienstes aufscheinen. Diese Bewohner*innen werden nicht als Klient*innen adressiert, weil sie ihre Bedarfe nicht explizit zum Ausdruck bringen und schlicht nicht gesehen werden und auch, weil die institutionellen Abläufe es nicht ermöglichen, die Bedarfe der Bewohner*innen jederzeit und gleichermaßen zu erkennen. Im Rahmen dieser Praktiken des Übersehens erfolgt eine zwischenzeitliche Exklusion aus dem Hilfesystem der Sozialen Arbeit, die aber prinzipiell jederzeit wieder aufgehoben werden kann. Nicht unbedingt besteht hierbei die Gefahr zur Exklusion aus der Gemeinschaft, wie dies bei Praktiken der Verweigerung der Fall ist. Durch Praktiken des Übersehens werden Bewohner*innen konstruiert, die aus verschiedenen Gründen ihren Hilfebedarf nicht anmelden und/oder recht passiv darauf warten, dass sie aufgesucht und als Klient*innen adressiert werden. Diese Praktiken können während aller Phasen - während des Ankommens, der Institutionalisierung, des Hilfebedarfs, des Abschieds - und wiederholt auftreten.

Beide Formen von undoing clientification verdeutlichen eine Art In-Balance: Entweder lehnen Bewohner*innen die Angebote der Sozialen Arbeit bzw. die Adressierung als Klient*in aktiv ab und Mitarbeitende des Sozialen Dienstes intervenieren umso häufiger. Oder die Bewohner*innen verhalten sich zu passiv bzw. äußern nicht ihren Hilfebedarf und Mitarbeitende des Sozialen Dienstes übersehen sie zwischenzeitlich. Als materieller Teilnehmer an diesen zwei Formen des undoing clientification spielt das eigene Zimmer eine wichtige Rolle: Dieser Privatraum fungiert bei der Verweigerung als Möglichkeit, sich vor den Angeboten des Sozialen Dienstes zu verschließen, andererseits kann er bei der Kontaktaufnahme mit dem Sozialen Dienst eine Hürde sein.

Eine dritte Art, um den Klient*innenstatus zu dekonstruieren, ist ebenfalls an Hirschauers Verständnis von ,undoing“ angelehnt. Unter Praktiken des Wartens werden in allen Phasen all jene ,doings and sayings“ zusammengefasst, die sich zum einen auf einen Leerlauf zwischen tagesstrukturierenden Aktivitäten (z. B. Mahlzeiten), Einzel- und Gruppenangeboten (z.B. Gedächtnistraining) oder auf andere Tätigkeiten (z.B. Gespräche) beziehen, und damit auf eine Infrastruktur, die sich aus dem zeitlichen Ablauf im Seniorenheim ergibt. Nach der aktiven Teilnahme etwa an einem Angebot zum Gedächtnistraining werden Klient*innen wieder zu Bewohner*innen und zugleich zu potenziellen Klient*innen, wenn sie ein später am Tag stattfindendes Angebot des Sozialen Dienstes in Anspruch nehmen wollen. Im Übergang vom Klient*innen- zum Bewohner*innenstatus erfolgen keine Adressierungen als Klient*in; diese werden nicht erwartet, angeboten oder abgelehnt, sie sind in diesen Momenten schlicht irrelevant. Diese Praktiken des Wartens sind durch das Sitzen auf Stühlen, Bänken oder Rollatoren gekennzeichnet, und es 
war häufiger zu beobachten, dass das Warten zwar in einer Gruppe von mehreren Personen vollzogen wurde, jedoch waren diese Personen dabei häufig nicht direkt im Gespräch miteinander. Zum anderen sind mit Praktiken des Wartens Leerläufe in Gesprächen zwischen Mitarbeitenden des Sozialen Dienstes und Klient*innen gemeint, die z.B. durch das Klingeln des Telefons unvermittelt entstehen. Dabei wird der Klient*innenstatus durch die Nichtadressierung des Gegenübers als Klient*in kurzzeitig neutralisiert (vgl. auch Schröder 2010). Als Aufgabe ergibt sich für die Mitarbeitenden des Sozialen Dienstes, diese potenziellen Klient*innen durch entsprechende Ansprache im Warten so zu adressieren, dass sie bereit sind, den Klient*innenstatus wieder anzunehmen.

\section{Zum Klient*innenstatus in der stationären Sozialen Altenarbeit: Schlussfolgerungen}

Ziel des Beitrags war es, über Konstruktions- und Dekonstruktionsprozesse den Klient*innenstatus in der stationären Sozialen Altenarbeit näher zu bestimmen und daraus den Auftrag der Sozialen Arbeit und Aufgaben der Mitarbeitenden des Sozialen Dienstes abzuleiten. Um solche Konstruktions- und Dekonstruktionsprozesse zu analysieren, wurde die Heuristik des un/doing clientification entwickelt, die es ermöglicht, eine konsequente Blickwinkelverschiebung von Menschen, die den Status als Klient*in interaktiv zuweisen (vgl. hierzu die konversationsanalytischen Studien von Bittner 1981; Meier und Wolff 1997; Meßmer 2013; Meßmer und Hitzler 2007; Reitemeier 2000) ${ }^{6}$, hin zu den sozialen Praktiken und ihren materiellen Teilnehmenden vorzunehmen, in denen der Klient*innenstatus im praktischen Tun hervorgebracht, aufrechterhalten, in Frage gestellt oder irrelevant gemacht wird. Die Analyse des empirischen Materials, das in Seniorenheimen erhoben wurde, verdeutlicht, dass die Konstruktion und Dekonstruktion des Klient*innenstatus durch spezifische „doings“ und ,sayings“ erfolgt, also im situativen praktischen Zusammenspiel und in gut aufeinander abgestimmten Choreographien, in die Bewohner*innen, Mitarbeitende, Angehörige, Alltagsgegenstände, technische Artefakte und Räume involviert sind. Dadurch konnte gezeigt werden, dass in den untersuchten Seniorenheimen Praktiken der Informationsvermittlung, der Integration, der Anähnlichung, der Herstellung von Hilfebedarf und der Erinnerung vollzogen werden, in denen Bewohner*innen als Klient*innen konstruiert werden, und dass wiederum Klient*innen durch die Praktiken der Verweigerung, des Übersehens und des Wartens als Bewohner*innen dekonstruiert werden können. Im alltäglichen Geschehen können sich diese Praktiken auch überlappen, was zu Widersprüchlichkeiten und Uneindeutigkeiten führen kann. Die Darstellung dieser Praktiken macht auch deutlich, dass der Status als Klient*in nicht als eine dauerhafte, unveränderliche Zuschreibung zu verstehen ist, sondern dass dieser Status situativ immer wieder neu zwischen den Teilnehmenden auszuhandeln und als solcher zu bestätigen ist. Diese alltägliche Aushandlungsarbeit wird durch eine prozesshafte Lebenslaufperspektive flankiert, weil die Bewohner*innen konti-

\footnotetext{
6 Siehe hierzu auch die Studien zur „,membership categorization analysis“ von Widdicombe (1998) und Lepper (2000), die sich aber nicht auf den Klient*innenstatus in der Sozialen Arbeit beziehen.
} 
nuierlich älter werden und sich im Laufe der Zeit ihre Bedarfe verändern können (vgl. Höppner und Wanka 2021); demzufolge können sich auch die Möglichkeiten in der alltäglichen interaktiven Aushandlungsarbeit verändern. Hinzu kommt, dass die Diversität der Bewohner*innen es verunmöglicht, von der einen Klientin oder dem einen Klienten auszugehen. Um als Mitarbeitende*r des Sozialen Dienstes mit dieser Diversität angemessen umgehen zu können, ergibt sich die Notwendigkeit zur flexiblen Aushandlungsarbeit und zur steten Reflexion von Vorannahmen und Zuschreibungen (vgl. Ammann 2020).

In der Konstruktion und Dekonstruktion des Klient*innenstatus wurden bestimmte Wissensbestände über Klient*innen der Sozialen Altenarbeit zum Ausdruck gebracht. Das Spektrum reicht von Vorstellungen, die Ideen zum aktiven Altern (vgl. WHO 2002) reproduzieren, bis hin zu Vorstellungen, die Alter mit Abbau, Funktionsverlust und Tod in Verbindung bringen (vgl. Cumming und Henry 1961). Mit diesen unterschiedlichen Vorstellungen zum Klient*innenstatus werden auch Bezüge zu den Differenzkategorien Alter, Krankheit und Behinderung hergestellt. Hier zeigt sich einmal mehr, dass Soziale Arbeit über Differenzkategorien operiert, d.h. ihre Arbeit darüber strukturiert (vgl. Streck et al. 2018, S. 243-248), und Menschen dadurch zugleich kategorisiert (vgl. Koch et al. 2018), wenn auch in dieser Untersuchung nicht entlang der in der Intersektionalitätsforschung klassischen Trias aus „race, class und gender“ (vgl. Crenshaw 1991; Lutz und Wenning 2001). Dies mag in organisatorischen und institutionellen Rahmenbedingungen, wie zum Beispiel den Einzugsvoraussetzungen wie der Pflegestufe, und dem damit in Zusammenhang stehenden recht allgemeinen Auftrag des Sozialen Dienstes in diesem Handlungsfeld zur Förderung von sozialer Teilhabe, Wohlbefinden, Schutz und Lebenszufriedenheit begründet liegen (vgl. Schweppe 2012). Die Vergabe des Klient*innenstatus brachte insofern Klarheit bei den Mitarbeitenden des Sozialen Dienstes, als sie dadurch ihren Zuständigkeitsbereich bzw. Auftrag spezifizieren konnten, der von Informationsvermittlung, über Formen der unterstützten Vergemeinschaftung bis hin zu palliativen Angeboten reichen kann. Die Vielfalt der in den Seniorenheimen zur Verfügung stehenden Angebote des Sozialen Dienstes und auch der methodischen Ausrichtung der Arbeit von Mitarbeitenden verdeutlicht zugleich deren Bedeutung in den untersuchten Seniorenheimen: Es sind vor allem die Mitarbeitenden des Sozialen Dienstes, die durch ihre Angebote die Bewohner*innen individuell unterstützen können und maßgeblich dazu beitragen, die Gemeinschaft in Balance zu halten.

Während in Studien oft von einer chronologisch ablaufenden Form der Klientifizierung mit bestenfalls daran anschließender Deklientifizierung ausgegangen wird (vgl. Meßmer 2013), zeigt dieser Beitrag, dass die Konstruktion bzw. Dekonstruktion des Klient*innenstatus im Handlungsfeld der stationären Sozialen Altenarbeit flexibler ist, d.h. nicht per se in einer chronologischen Abfolge verläuft. Die Erkenntnisse zum undoing clientification verdeutlichen etwa, dass die Praktiken der Verweigerung, des Übersehens und des Wartens in verschiedenen Phasen auftreten können, was einen zirkulären Prozess der Konstruktion und Dekonstruktion des Klient*innenstatus nahelegt. Gleichwohl flankieren Prozesse der Konstruktion und Dekonstruktion des Klient*innenstatus lediglich die Hervorbringung des Bewohner*innenstatus. Der Bewohner*innenstatus wird im Gegensatz zum Klient*innenstatus kontinuierlicher und stabiler hervorgebracht, d.h. im Seniorenheim 
lebende Menschen sind zuallererst Bewohner*innen und nicht per se und dauerhaft auch Klient*innen der Sozialen Arbeit. Dies könnte zusätzlich zur engen Verzahnung von Sozialem Dienst mit den alltagsweltlichen Zusammenhängen, dem nicht immer klar definierten Auftrag von Sozialer Arbeit und dem fehlenden Wissen zur Funktion von Sozialer Arbeit im Seniorenheim seitens einiger Bewohner*innen ein weiterer Grund dafür sein, dass das Handlungsfeld der stationären Sozialen Altenarbeit bisher noch relativ unbestimmt ist. $\mathrm{Zu}$ fragen wäre, ob das Merkmal der Zirkularität des Klient*innenstatus allein für das untersuchte stationäre Setting von Bedeutung ist, oder ob sich in anderen stationären Settings der Sozialen Arbeit Parallelen zeigen.

Der Beitrag verdeutlicht schließlich, wie der Klient*innenstatus im Handlungsfeld der stationären Sozialen Altenarbeit jenseits von Soll-Vorstellungen im praktischen Tun gemacht wird: Sowohl die ihm inhärente Zirkularität als auch die Erkenntnis, dass der Klient*innenstatus nicht allein ein interaktives Ko-Produkt von und für Menschen ist, betont den Stellenwert von materialitätsbezogenen Ansätzen, wie sie seit Kurzem in der Altersforschung diskutiert werden (vgl. Höppner 2021; Höppner und Urban 2018). Eine materialitätsbezogene Perspektive kann nicht nur die Theoriebildung Sozialer Arbeit befruchten, sondern auch dazu beitragen, praktisches Handeln auf andere Art und Weise zu reflektieren.

Funding Open Access funding enabled and organized by Projekt DEAL.

Open Access Dieser Artikel wird unter der Creative Commons Namensnennung 4.0 International Lizenz veröffentlicht, welche die Nutzung, Vervielfältigung, Bearbeitung, Verbreitung und Wiedergabe in jeglichem Medium und Format erlaubt, sofern Sie den/die ursprünglichen Autor(en) und die Quelle ordnungsgemäß nennen, einen Link zur Creative Commons Lizenz beifügen und angeben, ob Änderungen vorgenommen wurden.

Die in diesem Artikel enthaltenen Bilder und sonstiges Drittmaterial unterliegen ebenfalls der genannten Creative Commons Lizenz, sofern sich aus der Abbildungslegende nichts anderes ergibt. Sofern das betreffende Material nicht unter der genannten Creative Commons Lizenz steht und die betreffende Handlung nicht nach gesetzlichen Vorschriften erlaubt ist, ist für die oben aufgeführten Weiterverwendungen des Materials die Einwilligung des jeweiligen Rechteinhabers einzuholen.

Weitere Details zur Lizenz entnehmen Sie bitte der Lizenzinformation auf http://creativecommons.org/ licenses/by/4.0/deed.de.

\section{Literatur}

Aghamiri, K., \& Streck, R. (2018). Ausgestalten, Bespielen, Verändern. Wie Adressat_innen Soziale Arbeit herstellen. In K. Aghamiri, A. Reinecke-Terner, R. Streck \& U. Unterkofler (Hrsg.), Doing Social Work. Ethnografische Forschung als Theoriebildung. Rekonstruktive Forschung in der Sozialen Arbeit (S. 107-130). Opladen: Barbara Budrich.

Aghamiri, K., Reinecke-Terner, A., Streck, R., \& Unterkofler, U. (Hrsg.). (2018). Doing Social Work. Ethnografische Forschung als Theoriebildung. Rekonstruktive Forschung in der Sozialen Arbeit. Opladen: Barbara Budrich.

Ammann, E. S. (2020). Diversität im stationären Pflegealltag. In K. Aner \& U. Karl (Hrsg.), Handbuch Soziale Arbeit und Alter (S. 251-261). Wiesbaden: Springer.

Ammann, E. S., Rauber, G., \& Salis Gross, C. (2019). Doing death' the Mediterranean way: end-of-life in a segregated nursing home. Mortality. https://doi.org/10.1080/13576275.2018.1483906.

Backes, G. M., \& Clemens, W. (2013). Lebensphase Alter. Eine Einführung in die sozialwissenschaftliche Alternsforschung (4. Aufl.). Weinheim, Basel: Beltz Juventa. 
Bittner, U. (1981). Ein Klient wird „gemacht“. Ergebnisse einer empirischen Untersuchung zur Struktur von Erstgesprächen in einer Erziehungsberatungsstelle. In E. v. Kardorff \& E. Koenen (Hrsg.), Psyche in schlechter Gesellschaft. Zur Krise klinisch psychologischer Tätigkeit (S. 103-137). München: Urban \& Schwarzenberg.

Böhringer, D., \& Wolff, S. (2012). Der PC als „Partner“ im institutionellen Gespräch. Zeitschrift für Soziologie, 39(3), 233-252.

Breidenstein, G., Hirschauer, S., Kalthoff, H., \& Nieswand, B. (2013). Ethnografie. Die Praxis der Feldforschung. Konstanz: UVK.

Butler, J. (2004). Undoing gender. New York: Routledge.

Crenshaw, K. (1991). Mapping the margins: Intersectionality, identity politics, and violence against women of color. Stanford Law Review, 43(6), 1241-1299.

Cumming, E., \& Henry, W. (1961). Growing old: the process of disengagement. New York: Basic Books.

Depner, A. (2015). Dinge in Bewegung. Zum Rollenwandel materieller Objekte. Eine ethnographische Studie über den Umzug ins Altenheim. Bielefeld: transcript.

Deutsch, F. M. (2007). Undoing gender. Gender \& Society, 21(1), 106-127.

Hall, S., Juhila, K., Matarese, M., \& van Nijnatten, C. (Hrsg.). (2014). Analysing social work communication. Discourse in practice. London,, New York: Routledge.

Hall, S., Juhila, K., Parton, N., \& Pösö, T. (Hrsg.). (2003). Constructing clienthood in social work and human services. London, New York: Jessica Kingsley Publishers.

Heitmann-Möller, A., \& Remmers, H. (2017). Pflegebett und Agency. Eine Untersuchung aus der Perspektive der Akteur-Netzwerk-Theorie von Bruno Latour. In L. Artner \& al (Hrsg.), Pflegedinge. Materialitäten in Pflege und Care (S. 133-162). Bielefeld: transcript.

Hirschauer, S. (2001). Das Vergessen des Geschlechts. Zur Praxeologie einer Kategorie sozialer Ordnung. Kölner Zeitschrift für Soziologie und Sozialpsychologie, 41, 208-236.

Hirschauer, S. (2013). Die Praxis der Geschlechter(in)differenz und ihre Infrastruktur. In J. Graf, K. Ideler \& S. Klinger (Hrsg.), Geschlecht zwischen Struktur und Subjekt. Theorie, Praxis, Perspektiven (S. 153-171). Opladen: Barbara Budrich.

Hirt, R. (2008). Soziale Arbeit in stationären Einrichtungen der Altenhilfe. In K.-A. Chassé \& H.-J. v. Wensierski (Hrsg.), Praxisfelder der sozialen Arbeit. Eine Einführung (S. 229-244). Weinheim, München: Juventa.

Höppner, G. (2021 im Erscheinen). Verteiltes Alter(n): Grundlagen einer materialitätstheoretisch informierten Kulturgerontologie. In F. Kolland, V. Gallistl \& V. Parisot (Hrsg.), Kulturgerontologie - Kulturalistische Perspektiven auf das Alter(n) im deutschsprachigen Raum. Wiesbaden: Springer VS.

Höppner, G., \& Brinkmann, A.-S. (2020). Die Funktionen von Dingen in der Lebenswelt von Adressat_innen und Konsequenzen für Soziale Arbeit. Empirische Befunde am Beispiel der stationären Sozialen Altenarbeit. Neue Praxis, 5, 442-457.

Höppner, G., \& Urban, M. (2018). Where and how do aging processes take place in everyday life? Answers from a new materialist perspective. Frontiers in Sociology. https://doi.org/10.3389/fsoc.2018.00007.

Höppner, G., \& Wanka, A. (2021). un/doing age. Multiperspektivität als Potential einer intersektionalen Betrachtung von Differenz- und Ungleichheitsverhältnissen. Zeitschrift für Soziologie, 50(1), 42-57.

Juhila, K., Pösö, T., Hall, C., \& Parton, N. (2003). Introduction. In C. Hall, K. Juhila, N. Parton \& T. Pösö (Hrsg.), Constructing clienthood in social work and human services (S. 11-24). London, New York: Jessica Kingsley Publishers.

Koch, M., Piñeiro, E., \& Pasche, N. (2018). Ambivalentes Differenzieren. Ein ethnografischer Blick auf organisationales (Un)Doing Ethnicity in einem Schweizer Jugendamt. In K. Aghamiri, A. Reinecke-Terner, R. Streck \& U. Unterkofler (Hrsg.), Doing Social Work. Ethnografische Forschung als Theoriebildung. Rekonstruktive Forschung in der Sozialen Arbeit (S. 131-150). Opladen: Barbara Budrich.

Kutscher, N., Ley, T., Seelmeyer, U., Siller, F., Tillmann, A., \& Zorn, I. (2020). Handbuch Soziale Arbeit und Digitalisierung. Weinheim, Basel: Juventa.

Lepper, G. (2000). Categories in text and talk. A practical introduction to categorization analysis. London: SAGE.

Lutz, H., \& Wenning, N. (2001). Unterschiedlich verschieden. Differenz in der Erziehungswissenschaft. Opladen: Leske + Budrich.

Meier, C., \& Wolff, S. (1997). Konversationsanalyse als Supervision. Über Gesprächsbeendigungen in helfenden Beziehungen. In G. Jakob \& H.-J. v. Wensierski (Hrsg.), Rekonstruktive Sozialpädagogik. Konzepte und Methoden sozialpädagogischen Verstehens in Forschung und Praxis (S. 161-176). Weinheim, München: Juventa. 
Messmer, H. (2013). Mikrophysiken der Wirklichkeitsproduktion - Institutionelle Praxis in der Sozialen Arbeit aus Sicht der ethnomethodologischen Konversationsanalyse. In G. Graßhoff (Hrsg.), Adressaten, Nutzer, Agency (S. 317-340). Wiesbaden: Springer VS.

Meßmer, H., \& Hitzler, S. (2007). Die soziale Produktion von Klienten - Hilfeplangespräche in der Kinderund Jugendhilfe. In W. Ludwig-Mayerhofer, O. Behrend \& A. Sondermann (Hrsg.), Fallverstehen und Deutungsmacht. Akteure der Sozialverwaltung und ihre Klienten (S. 41-74). Wiesbaden: Barbara Budrich.

Ratzenböck, B. (2017). Recycelte Fernseher und ,abgestochene' Computer. Zur Erforschung von Medienerfahrungen von Frauen 60+ durch Interaktion mit Medienobjekten während, Walking Interviews“ in Wohnräumen. In I.C. Endter \& S. Kienitz (Hrsg.), Alter(n) als soziale und kulturelle Praxis. Ordnungen, Beziehungen, Materialitäten (S. 245-264). Bielefeld: transcript.

Reckwitz, A. (2003). Grundelemente einer Theorie sozialer Praktiken: Eine sozialtheoretische Perspektive. Zeitschrift für Soziologie, 32(4), 282-301.

Reitemeier, U. (2000). Zum interaktiven Umgang mit einbürgerungsrechtlichen Regelungen in der Aussiedlerberatung: gesprächsanalytische Beobachtungen $\mathrm{zu}$ einem authentischen Fall. Zeitschrift für qualitative Bildungs-, Beratungs- und Sozialforschung, 1(2), 253-282.

Schäfer, H. (2016). Grundlagen, Rezeption und Forschungsperspektive der Praxistheorie. In H. Schäfer (Hrsg.), Praxistheorie. Ein soziologisches Forschungsprogramm (S. 9-24). Bielefeld: trancript.

Schatzki, T. R. (2002). The site of the social. A philosophical account of the constitution of social life and change. Pennsylvania: Pennsylvania State University Press.

Schatzki, T. R. (2016). Praxistheorie als flache Ontologie. In H. Schäfer (Hrsg.), Praxistheorie. Ein soziologisches Forschungsprogramm (S. 29-44). Bielefeld: transcript.

Schmidt, T. R. (2012). Soziologie der Praktiken - Konzeptionelle Studien und empirische Analysen. Berlin: Suhrkamp.

Schröder, J. (2010). „Es klingelt“ - Institutionelle Kommunikation in sozialen Diensten. Neue Praxis, 5 , 493-512.

Schweppe, C. (Hrsg.). (1996). Soziale Altenarbeit. Pädagogische Arbeitsansätze und die Gestaltung von Lebensentwürfen im Alter. Weinheim, München: Juventa.

Schweppe, C. (2012). Soziale Altenarbeit. In W. Thole (Hrsg.), Grundriss Soziale Arbeit (S. 505-521). Wiesbaden: VS.

Strauss, A.L. (1998). Grundlagen qualitativer Sozialforschung: Datenanalyse und Theoriebildung in der empirischen und soziologischen Forschung. Stuttgart: UTB.

Streck, R., Aghamiri, K., Unterkofler, U., \& Reinecke-Terner, A. (2018). Was kennzeichnet Soziale Arbeit? - Elemente einer Theorie des Doing Social Work. In K. Aghamiri, A. Reinecke-Terner, R. Streck \& U. Unterkofler (Hrsg.), Doing Social Work. Ethnografische Forschung als Theoriebildung. Rekonstruktive Forschung in der Sozialen Arbeit (S. 237-259). Opladen: Barbara Budrich.

Unterkoffler, U., Reinecke-Terner, A., Streck, R., \& Aghamiri, K. (2018). Einleitung: Doing Social Work - Ethnografische Forschung als Theoriebildung. In K. Aghamiri, A. Reinecke-Terner, R. Streck \& U. Unterkofler (Hrsg.), Doing Social Work. Ethnografische Forschung als Theoriebildung. Rekonstruktive Forschung in der Sozialen Arbeit (S. 7-20). Opladen: Barbara Budrich.

West, C., \& Zimmerman, D.H. (1987). Doing gender. Gender \& Society, 1(2), 125-151.

Widdicombe, S. (1998). 'But you don't class yourself': the interactional management of categoy membership and non-membership. In C. Antake \& S. Widdicombe (Hrsg.), Identities in talk (S. 52-70). London: SAGE.

World Health Organisation (2002). Active ageing: a policy framework. Geneva: WHO. 